\title{
DESEMPEÑO SÍSMICO DE UN CENTRO DE SALUD DE TRES NIVELES CON O SIN AISLADORES
}

\author{
SEISMIC PERFORMANCE OF A THREE-LEVEL HEALTH CENTER WITH OR \\ WITHOUT INSULATORS
}

PABLO CESAR ARCAYA PANCCA 1

\section{Resumen}

El presente trabajo trata sobre el desempeño sísmico de un edificio (centro de salud) de tres niveles con o sin aisladores, el cual tiene como objetivos conocer la metodología aplicada, analizar los resultados obtenidos y establecer consideraciones que se deben tener en cuenta en el diseño de los aisladores. El problema a medir fue el desempeño sísmico en un centro de salud de tres niveles con o sin aisladores, siendo los tipos de aisladores a considerar tres tipos: aisladores de alto amortiguamiento (HDR), aisladores con núcleo de plomo (LRB) y sistema de péndulo friccional (FPS). La metodología utilizada fue aplicando la normatividad chilena NCh2745-2003, el mismo que es una adaptación del "código internacional para edificios" (IBC), por sus siglas en ingles que se basa en una norma americana para el diseño de aisladores y a través del modelado de la estructura con una programa computacional como es el SAP 2000.Los resultados obtenidos fueron para una estructura sin aislación. Del primer al último nivel se tiene valores de $0.35,0.34$ y $0.29 \mathrm{seg}$. de periodo fundamental, para el caso con aisladores de alto amortiguamiento se obtuvieron los valores de $2.98,2.97,2.56$ de periodo fundamental, para aisladores con núcleo de plomo los valores fueron de $2.76,2.75$ y 2.38 y para aisladores con sistema de péndulo friccional los valores eran $1.24,1.22$ y 1.06 respectivamente. Al comparar el desempeño sísmico de una estructura es mejor con aisladores, en comparación con una estructura sin aisladores, siendo los aisladores de alto amortiguamiento de mejor desempeño sísmico.

Palabras clave: Aisladores, edificio

\section{Abstract}

The present work deals with the seismic performance of a building (health center) of three levels with or without insulators, which aims to know the methodology applied, analyze the results obtained and establish considerations that must be taken into account in the design of the insulators. The problem to be measured was the seismic performance in a three-level health center with or without insulators, with the types of insulators to be considered three types: highdamping insulators (HDR), lead-core insulators (LRB) and frictional pendulum (FPS). The methodology used was applying the Chilean regulation NCh2745-2003, the same that is an adaptation of the "international code for buildings" (IBC), for its acronym in English "which is based on an American standard for the design of insulators and through the modeling of the structure with a computational program such as SAP 2000. The results obtained were for an uninsulated structure, from the first to the last level it has values of $0.35,0.34$ and $0.29 \mathrm{sec}$. of fundamental period, for the case with high damping insulators the values of 2.98, 2.97, 2.56 of fundamental period were obtained, for insulators with lead core the values were 2.76 , 2.75 and 2.38 and for insulators with frictional pendulum system the values were 1.24, 1.22 and 1.06 respectively. When comparing the seismic performance of a structure it is better with insulators, in comparison with a structure without insulators, being the insulators of high damping of better seismic performance.

Keywords: Insulators, building

1 MAESTRO EN INGENIERÍA CIVIL CON MENCIÓN EN ESTRUCTURAS 
La sismicidad en el Perú a través del tiempo, nos ha mostrado la ocurrencia de grandes sismos destructivos que ocurren desde el siglo pasado hasta la actualidad, el cual ha originado grandes pérdidas humanas y materiales, el mismo que debe tomarse en cuenta en aplicar nuevas tecnologías, como es el uso de aisladores sísmicos en las estructuras.La zona sur del Perú, se ubica en el cinturón de fuego del Pacifico, entre la placa de Nazca y la placa sudamericana, el cual es propenso a la ocurrencia de eventos sísmicos, dentro de los cuales están las edificaciones esenciales como son los centros de salud. Los centros de salud, son edificaciones esenciales que tienen que seguir funcionando aun después de ocurrido un evento sísmico, y no debe de colapsar, a fin de brindar resguardo a las personas.

Asimismo, una de las novedades en el desempeño sísmico de las estructuras, es el uso de aisladores sísmicos en la base. Los aisladores son elementos que separan una estructura del suelo para reducir los efectos del sismo, ya que absorben las deformaciones que produce un evento sísmico sobre una estructura. La mayoría de los edificios aislados en el mundo tienen un buen desempeño sísmico en las estructuras. (Arriagada, Jaime, 2005). El aislamiento sísmico es una alternativa para el buen desempeño de la estructura frente a estos eventos. (Leigh Méndez, Patricio,2002). Esta técnica consiste en la separación sueloestructura, independizando así a la estructura del movimiento que se propaga a través del suelo en donde esta cimentada, permitiendo un desempeño sísmico adecuado. (Castillo et al., 2011). La protección con aisladores en edificaciones es una fase fundamental en el desempeño de estructuras especialmente cuando se produce en países en desarrollo (Symans, M. (1998). La aislación de edificaciones consiste en colocar una interfase flexible entre el suelo y la estructura de forma que se reduzcan considerablemente las solicitaciones sísmicas (Korswagen, et al. 2012). En este contexto cabe considerar que las edificaciones de salud son de vital importancia después de producido los eventos sísmicos (Azabache, J. et al. (2014). El desempeño se cuantifica en términos de la cantidad de daño en un edificio afectado por un movimiento sísmico y el impacto que tienen estos daños en las actividades posteriores al evento (Bertero, 2010).

La mayoría de las metodologías de evaluación sólo consideran un nivel de movimiento del terreno (sismo severo, como el sismo del 23 de junio del 2001, en Perú), para el cual la edificación no debería colapsar, obviando la posibilidad que puedan ocurrir daños y pérdidas importantes asociadas a sismos de naturaleza más frecuente. (Bertero, 2010). Los sismos severos o terremotos, son los eventos naturales de súbita liberación casi instantánea de energía acumulada en el interior de la Tierra. Esta liberación se manifiesta en su forma más general por el movimiento diferencial de bloques a lo largo de fallas: (Izaguirre Corona, A. M, 2007). La región sur-occidental del Perú está situada en la zona de subducción de la placa Nazca y la placa Sudamericana. Ésta es una zona de alta actividad sísmica en donde, de acuerdo a la sismicidad histórica, han ocurrido sismos severos en la escala de Richter.

El departamento de Tacna siempre ha sido una encrucijada puesto que han ocurrido movimientos sísmicos que sucedieron en los años $1604,1615,1784,1883$ y 1868, los cuales trajeron consigo una destrucción y asolamiento de la región. Después de muchos años ocurrió un sismo de gran magnitud el 23 de junio del 2001 , de 6.9 grados en la escala de Richter, siendo su epicentro a $20 \mathrm{Km}$ de la ciudad de Ocoña, a la altura de Ático (Arequipa). En el departamento de Tacna los damnificados y afectados suman alrededor de 74109 personas (INDECI, 2001).

El aislamiento de base es desacoplar al edificio o estructura de los componentes horizontales del movimiento del suelo, interponiendo elementos estructurales con rigidez horizontal baja entre la estructura y la fundación. Esto permite "ablandar" la respuesta sísmica de la estructura logrando una frecuencia fundamental que es mucha más baja que su frecuencia como base fija y las frecuencias predominantes del movimiento del suelo (Arriagada, 2005). El sistema de aislación es el conjunto de elementos estructurales que incluye a todos los aisladores, sus conexiones y a los elementos estructurales que transmiten fuerza entre el sistema de aislación y la superestructura y subestructura. A la estructura que se encuentra encima del sistema de aislación se le denomina superestructura y a la que se encuentra debajo subestructura. La interfase de aislación es el límite imaginario entre la superestructura y la subestructura. Entre los principales tipos de aisladores tenemos a los: Aisladores elastoméricos, los aisladores con núcleo de plomo y el sistema de péndulo de fricción invertido y de doble curvatura. A través de los 
años se han desarrollado programas que permiten el análisis dinámico no lineal de sistemas de aislamiento (Naeim y Kelly, 1994): N-PAD, 3D-BASIS, ETABS y SAP2000. Los principales métodos de modelamiento referenciales para estructuras con aisladores sísmicos son Método cuasiestatico, el dinámico exacto y el de masa corregida (Romo et al, 2007). El sistema de aislamiento sísmico se diseña para reducir fuerzas y por tanto disminuir costos, pero la reducción lograda no tiene el mismo valor para fuerzas y para costos, una reducción de las fuerzas por un factor de 3 no reduce costos por la misma cantidad (Bohórquez y Gómez, 2009). Si la superestructura fue diseñada para los mismos niveles de ductilidad como para una estructura de base fija, entonces se tiene que la baja resistencia de la estructura disminuye la eficiencia del sistema (Bohórquez y Gómez, 2009).

\section{OBJETIVOS}

Describir la metodología empleada y analizar los resultados obtenidos del desempeño sísmico en el desempeño sísmico de un centro de salud de tres niveles con o sin aisladores.
Establecer consideraciones que se deben tener en cuenta en el desempeño sísmico de un centro de salud de tres niveles con o sin aisladores.

\section{METODOLOGÍA}

Investigación exploratoria, correlacional.El ámbito social corresponde a la zona 4 (según NTP E.030-2016) del sector urbano de la ciudad de Tacna. Comprende la comparación del desempeño sísmico de un centro de salud de tres niveles con o sin aisladores. La población está conformada por los tipos de aisladores sísmicos que se utilizan en la base de la estructura, y por la estructura sin aisladores, según:

Modelo 01: estructura de 3 niveles con pórticos de concreto armado sin aisladores sísmicos en la dirección $\mathrm{x}$ del sismo.

Modelo 02: estructura de 3 niveles con pórticos de concreto armado sin aisladores sísmicos en la dirección y del sismo.
Modelo 03: estructura de 3 niveles con pórticos de concreto armado y con aisladores sísmicos, tipo amortiguamiento alto (aisladores elastoméricos convencionales).

Modelo 04: estructura de 3 niveles con pórticos de concreto armado y con aisladores sísmicos, tipo núcleo de plomo.

Modelo 05: estructura de 3 niveles con pórticos de concreto armado y con aisladores sísmicos tipo péndulo friccional.

Se utilizaron los Softwares de diseño para los planos en planta y elevación (autocad) y Software de simulación de estructuras con y sin aisladores sísmicos.

\section{RESULTADOS}

Los resultados se presentan según modelos de estructura:

a. Modelo 01 de la Estructura

Una vez modelado y analizada la estructura, se obtuvieron los resultados que se muestran a continuación:

\begin{tabular}{|c|c|}
\hline Descripción & Base fija \\
\hline Periodo fundamental piso 1 & 0.35 \\
\hline Periodo fundamental piso 2 & 0.34 \\
\hline Periodo fundamental piso 3 & 0.29 \\
\hline
\end{tabular}

\begin{tabular}{|l|c|c|l|l|l|c|c|}
\hline \multirow{2}{*}{ DESCRIPCION } & \multicolumn{8}{|c|}{ DEFORMACIONES SISMICAS PARA SISMO EN X } \\
\cline { 2 - 8 } & $\mathrm{U} 1(\mathrm{~m})$ & $\mathrm{U} 2(\mathrm{~m})$ & $\begin{array}{l}\text { D.Rel } \\
\text { U1 }(\mathrm{m})\end{array}$ & $\begin{array}{l}\text { D.Rel } \\
\mathrm{U} 2(\mathrm{~m})\end{array}$ & $h(\mathrm{~m})$ & Drift en $\mathrm{X}$ & Condición \\
\hline PISO 1 & 0.00497 & 0.0000097 & 0.0000000 & -0.007660 & 3.25 & 0.0000000 & OK \\
\hline PISO 2 & 0.00497 & 0.0000097 & 0.0000000 & -0.005140 & 3.50 & 0.0000000 & OK \\
\hline PISO 3 & 0.00497 & 0.0000097 & 0.0049700 & 0.000058 & 3.50 & 0.0014200 & OK \\
\hline
\end{tabular}


b. Modelo 02 de la Estructura

\begin{tabular}{|c|c|}
\hline descripción & Base fija \\
\hline Periodo fundamental piso 1 & 0.35 \\
\hline Periodo fundamental piso 2 & 0.34 \\
\hline Periodo fundamental piso 3 & 0.29 \\
\hline
\end{tabular}

\begin{tabular}{|l|c|c|l|l|l|l|c|}
\hline \multirow{2}{*}{ DESCRIPCION } & \multicolumn{9}{|c|}{ DEFORMACIONES SISMICAS PARA SISMO EN Y } \\
\cline { 2 - 8 } & U1 $(\mathrm{m})$ & $\mathrm{U} 2(\mathrm{~m})$ & $\begin{array}{l}\text { D.Rel } \\
\text { U1 }(\mathrm{m})\end{array}$ & $\begin{array}{l}\text { D.Rel } \\
\text { U2 }(\mathrm{m})\end{array}$ & $\mathrm{h}(\mathrm{m})$ & Drift en $\mathrm{Y}$ & Condición \\
\hline PISO 1 & 0.0000497 & 0.0000097 & 0.0000000 & -0.00766 & 3.25 & 0.0000000 & OK \\
\hline PISO 2 & 0.0000497 & 0.0000097 & 0.0000000 & -0.00514 & 3.50 & 0.0000000 & OK \\
\hline PISO 3 & 0.0000497 & 0.0000097 & 0.0002820 & 0.000058 & 3.50 & 0.0000806 & OK \\
\hline
\end{tabular}

c. Modelo 03 deEstructura

\begin{tabular}{|c|c|c|}
\hline descripción & Base Fija & $\begin{array}{c}\text { Base } \\
\text { Aislada }\end{array}$ \\
\hline Periodo fundamental piso 1 & 0.35 & 2.98 \\
\hline Periodo fundamental piso 2 & 0.34 & 2.97 \\
\hline Periodo fundamental piso 3 & 0.29 & 2.56 \\
\hline
\end{tabular}

\begin{tabular}{|l|c|c|c|c|c|c|}
\hline & \multicolumn{3}{|c|}{ EJE X } & \multicolumn{3}{c|}{ EJE Y } \\
\cline { 2 - 7 } & $\begin{array}{c}\text { Base } \\
\text { Fija }\end{array}$ & $\begin{array}{c}\text { Base } \\
\text { Aislada }\end{array}$ & $\begin{array}{c}\text { \% de } \\
\text { reducción }\end{array}$ & $\begin{array}{c}\text { Base } \\
\text { Fija }\end{array}$ & $\begin{array}{c}\text { Base } \\
\text { Aislada }\end{array}$ & $\begin{array}{c}\text { \% de } \\
\text { reducción }\end{array}$ \\
\hline Cortante Basal (Ton) & 7.97 & 6.45 & 19.02 & 0.71 & 0.64 & 10.93 \\
\hline Momento flector (Ton-m) & 12.95 & 10.80 & 16.61 & 1.16 & 1.02 & 12.08 \\
\hline $\begin{array}{l}\text { Máx. Desplazamiento } \\
\text { Relativo a la base (cm) }\end{array}$ & 0.4970 & 0.3648 & 26.60 & 0.500 & 0.3691 & 26.18 \\
\hline $\begin{array}{l}\text { Máximo Desplazamiento } \\
\text { de entrepiso (cm) }\end{array}$ & 0.0010 & 0.0004 & 60.00 & 0.001 & 0.0004 & 60.00 \\
\hline
\end{tabular}

Un análisis de los resultados obtenidos permite apreciar el principal efecto del aislamiento sísmico en el comportamiento dinámico de la estructura que es el incremento del periodo fundamental de vibración. Este incremento es una consecuencia de la flexibilidad impuesta por los aisladores en el sistema estructural.

Se observa también que el porcentaje de reducción en el eje "Y" es menor que el Eje " $X$ ". Esto se debe principalmente a que la estructura en el eje " $Y$ " es mucho más rígido que el " $X$ ", y dado que la edificación aislada muestra un poco de efecto de vibración, como resultado de la cantidad finita de flexibilidad del superestructura.

Se puede apreciar la reducción de las fuerzas cortantes en la edificación aislada, en el eje " $X$ " se observa una reducción del cortante basal del $19.02 \%$ y en la dirección "Y" un $10.93 \%$ de reducción. El momento en la dirección " $X$ " se reduce en un $16.61 \%$ y en la dirección " $Y$ " en $12.08 \%$.

\section{d. Modelo 04 de la Estructura}

Una vez modelada analizada la estructura, se obtuvieron los resultados que se muestran a continuación:

\begin{tabular}{|l|c|c|}
\hline \multicolumn{1}{|c|}{ descripción } & Base Fija & Base Aislada \\
\hline Periodo fundamental piso 1 & 0.35 & 2.76 \\
\hline Periodo fundamental piso 2 & 0.34 & 2.75 \\
\hline Periodo fundamental piso 3 & 0.29 & 2.38 \\
\hline
\end{tabular}




\begin{tabular}{|l|c|c|c|c|c|c|}
\hline & \multicolumn{3}{|c|}{ EJE X } & \multicolumn{3}{c|}{ EJE Y } \\
\cline { 2 - 7 } & $\begin{array}{c}\text { Base } \\
\text { Fija }\end{array}$ & $\begin{array}{c}\text { Base } \\
\text { Aislada }\end{array}$ & $\begin{array}{c}\text { \% de } \\
\text { reducción }\end{array}$ & $\begin{array}{c}\text { Base } \\
\text { Fija }\end{array}$ & $\begin{array}{c}\text { Base } \\
\text { Aislada }\end{array}$ & $\begin{array}{c}\text { \% de } \\
\text { reducción }\end{array}$ \\
\hline Cortante Basal (ton) & 7.97 & 6.44 & 19.14 & 0.71 & 0.21 & 70.01 \\
\hline Momento flector (ton-m) & 12.95 & 10.94 & 15.49 & 1.16 & 0.70 & 40.01 \\
\hline $\begin{array}{l}\text { Máximo Desplazamiento } \\
\text { Relativo a la base (cm) }\end{array}$ & 0.4970 & 0.3140 & 36.83 & 0.5000 & 0.3167 & 36.67 \\
\hline \begin{tabular}{l} 
Máximo $\begin{array}{l}\text { Desplazamiento de } \\
\text { entrepiso (cm) }\end{array}$ \\
\hline
\end{tabular} & 0.0010 & 0.0002 & 80.00 & 0.0010 & 0.0002 & 80.00 \\
\hline
\end{tabular}

\section{e. Modelo 05 de la Estructura}

\begin{tabular}{|l|c|c|}
\hline \multicolumn{1}{|c|}{ descripción } & Base Fija & Base Aislada \\
\hline Periodo fundamental piso 1 & 0.35 & 1.24 \\
\hline Periodo fundamental piso 2 & 0.34 & 1.22 \\
\hline Periodo fundamental piso 3 & 0.29 & 1.06 \\
\hline
\end{tabular}

\begin{tabular}{|l|c|c|c|c|c|c|}
\hline & \multicolumn{3}{|c|}{ EJE X } & \multicolumn{3}{c|}{ EJE Y } \\
\cline { 2 - 7 } & $\begin{array}{c}\text { Base } \\
\text { Fija }\end{array}$ & $\begin{array}{c}\text { Base } \\
\text { Aislada }\end{array}$ & $\begin{array}{c}\text { \% de } \\
\text { reducción }\end{array}$ & $\begin{array}{c}\text { Base } \\
\text { Fija }\end{array}$ & $\begin{array}{c}\text { Base } \\
\text { Aislada }\end{array}$ & $\begin{array}{c}\% \text { de } \\
\text { reducción }\end{array}$ \\
\hline Cortante Basal (ton) & 7.97 & 6.52 & 18.14 & 0.71 & 0.08 & 88.94 \\
\hline Momento flector (ton-m) & 12.95 & 11.20 & 13.51 & 1.16 & 0.26 & 77.86 \\
\hline $\begin{array}{l}\text { Máximo Desplazamiento } \\
\text { Relativo a la base (cm) }\end{array}$ & 0.4970 & 0.062 & 87.49 & 0.5000 & 0.064 & 87.15 \\
\hline $\begin{array}{l}\text { Máximo Desplazamiento } \\
\text { de entrepiso (cm) }\end{array}$ & 0.0010 & 0.0002 & 80.00 & 0.0010 & 0.0002 & 80.00 \\
\hline
\end{tabular}

De los resultados obtenidos se muestra que los aisladores sísmicos influye en el comportamiento de la estructura, ya que se genera el incremento en el periodo fundamental de vibración, producto de la flexibilidad, que se obtiene de utilizar los aisladores en la estructura modelada.

Al analizar los resultados se observa la reducción de las fuerzas cortantes y del momento flector en la estructura. Aquí la disminución es más pareja dado que ninguno de los ejes presenta una rigidez considerable.

Al observar las respuestas de los resultados obtenidos, se ve como la estructura rígida, varia los valores de fuerzas cortantes y momentos flectores debido a su corto periodo de vibración. En las estructuras rígidas la amplificación de la aceleración del suelo (usualmente 2 a 3 veces) daña el funcionamiento de equipos y accesorios sensibles.

También se nota la disminución de los desplazamientos de entrepiso. Cabe notar que dado que el Sistema de Péndulo Friccional (FPS), las reducciones de las distorsiones angulares de entrepiso son considerables. Los Sistemas de Péndulo Friccional son los que mejor comportamiento exhibe en el control de rotaciones (Bozzo et al).

Los desplazamientos son menores que en la estructura inicial sin aisladores, dado que los sistemas deslizantes el coeficiente de fricción juega un papel importante. En este estructura igual que la anterior la mayor parte de este deslizamiento está concentrado en el sistema de aislamiento, a través de las deformaciones laterales de los aisladores.

Actualmente el Perú no cuenta con una metodología para ver el desempeño sísmico de una estructura, el cual la metodología que se utiliza es con la normativa chilena NCh27452003, el mismo que es una adaptación del "código internacional para edificios" (IBC). 


\section{DISCUSIÓN}

Se concluye que el desempeño sísmico de la estructura con aisladores es extremadamente diferente en comparación con la estructura sin aisladores. Se aplicó la metodología de $\mathrm{NCH} 2745-2003$ para el diseño de los aisladores, ya que el Perú no cuenta con una norma de edificaciones esenciales como los centros de salud. Se analizó que al comparar el desempeño sísmico de una estructura es mejor con aisladores, en comparación con una estructura sin aisladores, siendo los aisladores de alto amortiguamiento de mejor desempeño sísmico, según la norma de diseño sismorresistente. Se estableció consideraciones que se debe tener en cuenta, para el diseño de aisladores es no diseñar en un suelo blando, cerca de fallas geológicas, en estructuras irregulares. Se recomienda proponer una norma que permita diseñar estructuras con aisladores sísmicos en el Perú. Ha futuro las edificaciones esenciales como centros de salud, deben diseñarse con aisladores, especialmente en la zona sísmica del Perú (zona de costa, que forma parte de circulo de fuego, donde se producen eventos sísmicos). Tener en cuenta el desempeño sísmico de una estructura con aisladores, para las diferentes zonas de la costa del Perú, ya que los aisladores deben diseñarse en suelos rígidos, de alta capacidad portante, en estructuras regulares de 3 pisos a más. Asimismo, se sugiere tomar en cuenta las consideraciones para el tipo de aisladores a usar en una estructura como es una edificación esencial.

\section{REFERENCIAS BIBLIOGRÁFICAS}

ACI Perú. (2001). Normas Peruanas de Estructuras. $2 a$ ed. Lima-Perú.

Aguiar Falconí, R. (2008). Análisis sísmico de edificios. Capítulo 9 aisladores de base elastomericos. Escuela Politécnica del Ejército. Quito Ecuador.

Arriagada, J. A. (2005). Aislación sísmica de un edificio de oficinas de siete Pisos. Análisis comparativo de comportamiento y costos con un edificio Tradicional. Tesis de ingeniería. Valdivia, Universidad Austral de Chile, pag12.

Azabache, J. et al. (2014). Diseño estructural comparativo entre los sistemas dual y con aislamiento sísmico en la base para una clínica de 8 pisos en la ciudad de Trujillo. Universidad PrivadaAntenor Orrego.

Bertero, V. (2010). Nuevas tecnologías en la construcción y el diseño de estructuras antisísmicas.

Castillo, J. et al. (2011). Análisis estructural y económico de edificio de 30 pisos en la ciudad de Cartagena, mediante análisis estático y empleando aisladores sísmicos. Universidad de Cartagena.

Corporación de Desarrollo Tecnológico. (2010). Protección sísmica de estructuras. Documento Técnico Nro. 29. Cámara Chilena de la
Construcción. Santiago, Chile. Editorial Trama Impresores S.A.

Guillen Zambrano, J. E. (2012). Tendencias actuales en la construcción sismorresistente para edificios en hormigón armado. Universidad Politécnica de Madrid.

Korswagen, et al. (2012). Análisis y diseño de estructuras con aisladores sísmicos en el Perú. Pontificia Universidad Católica del Perú.

Leigh M., Patricio (2002). Aislación sísmica de estructuras en base a sistemas híbridos de goma y aleaciones: análisis, experimentación y diseño. Pontificia Universidad Católica de Chile.

Norma Técnica de Edificación (2011). E.020. Cargas. Reglamento Nacional de Edificaciones. Lima, Perú.

Romo, M. y Aguiar Falconi, R. y Bravo, V. (2007). Alternativas de modelamiento de estructuras con aisladores sísmicos mediante SAP 2000. II Congreso de Ciencia y Tecnología. Escuela Politécnica del Ejército. Quito, Ecuador.

Symans, M. (1998). Seismic protective systems: seismic isolation - Instructional Material Complementing FEMA451, Design Examples.

Presentado: Marzo 2017

Aceptado: Junio 2017 